\title{
Validation of the Mayo alliance prognostic system for mastocytosis
}

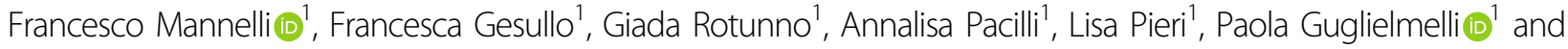 \\ Alessandro M. Vannucchi (i]
}

To the Editor:

Systemic mastocytosis (SM) is a myeloproliferative disorder characterized by extreme heterogeneity of clinical manifestations, course and prognosis. According to the WHO 2016 classification, selected clinical findings are useful for distinguishing aggressive from indolent variant, a distinction that drives therapeutic decisions ${ }^{1}$. Additional clinical and laboratory variables were then shown to correlate with survival, including advanced age, elevated beta-2-microglobulin and alkaline phosphatase (ALP) levels $^{2,3}$. Also, mutations of selected genes, usually associated with "high risk" features in other myeloid neoplasms $s^{4,5}$, were identified, that provide clinically relevant information about disease' course; in particular, mutation in SRSF2, ASXL1, RUNX1, and CBL were shown to be prognostically relevant ${ }^{6-8}$, and were also validated recently in our patients' cohort ${ }^{9}$. Conversely, unlike other myeloproliferative neoplasm, particularly myelofibrosis ${ }^{10}$, cytogenetic abnormalities in patients with SM are infrequent and have poor prognostic relevance ${ }^{11}$. By integrating the above prognostically relevant clinical and molecular parameters in a large cohort of SM patients seen at Mayo Clinic $(n=580)$, Pardanani et al. recently derived two prognostic models that may find wide clinical application: the first one was based upon clinical parameters only, the second enlisted both clinical and genomic findings ${ }^{9}$. Variables enlisted in the clinical model include SM variant (advanced versus indolent/smouldering SM), age $>60$ years, platelets $<150 \times 10^{9} / \mathrm{L}$, sexadjusted anemia and serum alkaline phosphatase above normal range, that are complemented by adverse

\footnotetext{
Correspondence: Alessandro M. Vannucchi (amvannucchi@unifi.it) ${ }^{1}$ CRIMM, Centro di Ricerca e Innovazione per le Malattie Mieloproliferative, Azienda Ospedaliera Universitaria Careggi, Dipartimento di Medicina Sperimentale e Clinica, Università degli Studi, Firenze, Italy
}

mutations (ASXL1, RUNX1, NRAS) in the hybrid clinicalmolecular score.

The aim of the current study was to assess the performance of the Mayo Alliance Prognostic System (MAPS) models in a real-life setting at our Center. After approval from the institutional review board, we interrogated our database for the availability of MAPS-related parameters, and finally applied the clinical-only model to 94 SM patients and the hybrid clinical-molecular model to 65 patients, out of a total of 127 patients with diagnosis of SM observed in the period from 2000 to 2018; information was last updated in August 2018.

Clinical and laboratory features of the study population are summarized in Table 1. According to WHO classification $^{1}, 82$ of $94(87.2 \%)$ patients were diagnosed with non-advanced forms, 78 (83.0\%) were indolent and 4 (4.3\%) smouldering SM. The 12 advanced cases included 9 aggressive variants (ASM, 9.6\%), 2 with an associated hematological neoplasm (SM-AHN, 2.1\%), and 1 mast cell leukemia (MCL, 1.1\%). As expected from other reports in larger cohorts, compared with advanced SM, the patients with indolent/smouldering SM were younger, had higher hemoglobin and platelet count, lower leukocyte count, and lower levels of serum alkaline phosphatase and tryptase. Overall, $98 \%$ of the patients were found positive for the KITD816V mutation. Additional myeloid mutations (ASXL1, RUNX1, and SRSF2) were assessed in 65 patients; for 21 of them, genotyping of NRAS was also available. Overall, we observed 6 cases with high risk mutations, all of which were included among advanced forms. The rate of adverse mutations (9.2\%) was lower that reported by Pardanani and colleagues $(21 \%)$, and is likely to be accounted for by a greater representation of advanced forms in their database. Fifty-eight percent of patients with advanced SM died, after a median follow-up of 23 months, as compared to $1.2 \%$ of indolent/ 
Table 1 Clinical and laboratory features of 94 patients with systemic mastocytosis (SM) included in the study

\begin{tabular}{|c|c|c|c|c|}
\hline Variables & Overall $n=94$ & Indolent/smouldering SM N=82 & Advanced SM N $=12$ & $p$ value \\
\hline Median age (range) & $47(17-80)$ & $45(17-80)$ & $64(23-78)$ & 0.01 \\
\hline Age > 60 years; $n(\%)$ & $26(27.7)$ & $19(23.2)$ & $7(58.3)$ & 0.017 \\
\hline Hemoglobin, g/dl, median (range) & $13.7(4.8-16.2)$ & $14.2(10.1-19.4)$ & $11.1(5.1-17.4)$ & $<0.001$ \\
\hline Anemia sex adjusted; $n$ (\%) & $22(23.4)$ & $11(13.4)$ & $11(91.7)$ & $<0.001$ \\
\hline Leukocyte count $\times 10^{9} / \mathrm{I}$, median (range) & $7.3(2.0-39.8)$ & $7.1(3.2-17.1)$ & $12.1(2.0-39.8)$ & 0.022 \\
\hline Platelet count $\times 10^{9} / l$, median (range) & $232(70-421)$ & $263(98-456)$ & $79(10-368)$ & $<0.001$ \\
\hline Platelet count $<150 \times 10^{9} /$; $n(\%)$ & $14(14.8)$ & $3(3.7)$ & $10(83.3)$ & $<0.001$ \\
\hline Serum tryptase $\mathrm{ng} / \mathrm{ml}$; median (range) & $33.0(3.1-7180)$ & $29.0(3.0-591)$ & $162.5(30.0-7180)$ & $<0.01$ \\
\hline Serum ALP, U/I; median (range) & $75(20-184)$ & $90(31-280)$ & $148.5(20-438)$ & 0.004 \\
\hline Serum ALP > UNL; $n(\%)$ & $21(22.3)$ & $14(17.1)$ & $7(58.3)$ & $<0.001$ \\
\hline KITD816V; $n$ (\%) $N=88$ & $86(97.7)$ & 75 (97.4) N Evaluable $=77$ & $11(100)$ N Evaluable $=11$ & 1.0 \\
\hline ASXL1 mutated; $n$ (\%) $N=65$ & $3(4.6)$ & 0 (0) $N$ Evaluable $=55$ & $3(30) \mathrm{N}$ Evaluable $=10$ & 0.003 \\
\hline RUNX1 mutated; $n(\%) N=65$ & $1(1.5)$ & $0(0) N$ Evaluable $=55$ & 1 (10) $N$ Evaluable $=10$ & 0.154 \\
\hline NRAS mutated; $n$ (\%) $N=21$ & 1 & $0(0) N$ Evaluable $=16$ & 1 (25) $N$ Evaluable $=5$ & 0.238 \\
\hline SRSF2 mutated; $n(\%) N=65$ & $1(1.5)$ & $0(0) N$ Evaluable $=55$ & 1 (10) $N$ Evaluable $=10$ & 0.154 \\
\hline Adverse mutations; $n$ (\%) $N=65$ & $6(9.2)$ & $0(0) N$ Evaluable $=55$ & 6 (50) $N$ Evaluable $=10$ & $<0.001$ \\
\hline Median follow-up in months (range) & $46(2.1-209)$ & $50.1(2.1-209)$ & $23.4(2.1-115)$ & 0.022 \\
\hline Deaths; $n(\%)$ & $8(8.5)$ & $1(1.2)$ & $7(58.3)$ & $<0.001$ \\
\hline
\end{tabular}

$S M$ systemic mastocytosis, $S M-A H N$ systemic mastocytosis with an associated hematological neoplasm, $A L P$ alkaline phosphatase, UNL upper normal limit, $N$ means the number of patients for which the particular information was available

$p$ values lower than significance threshold are highlighted by bold

smouldering SM, after a median follow-up of 50 months $(P<0.001)$.

Owing to the prevalence of indolent/smouldering cases in our cohort, we carried out a 3-tier stratification of patients according to the MAPS clinical and hybrid clinical-molecular model, by gathering patients with 1-2 and 3 to 5 risk factors in 2 categories only (intermediate and high risk, respectively) while patients with no risk factors were included in a low-risk category. Results of the analysis in our cohort excellently validated the performance of the MAPS score, overall resulting in a dramatic separation of patients with dismal overall survival (OS) and event-free survival (EFS) from others with very favorable course (Fig. 1). In particular, both models were able to identify an adverse risk category, with a median OS of only 18.8 months (95\% CI, 0.1-48), significantly shorter than the intermediate and low-risk categories (OS not reached in both) (Fig. 1a, c for the clinical-only and hybrid model, respectively); the lack of a significant difference between low- and intermediate-risk patients can reasonably be ascribed to the limited number of events in our cohort mainly including not aggressive forms of SM, as exemplified by separate analysis of indolent/smouldering cases (Fig. 1b, $\mathrm{d}$ for the 2 models)
We also evaluated performance of the Mayo models using event-free survival (EFS) as an endpoint; events were death and progression from indolent to aggressive variant, that constitutes a major clinical event influencing long term outcome in SM (Fig. 1e-h). As shown in Fig. 1, the clinical-only model allowed prediction of shorter EFS in the entire cohort $(P=0.053$; Fig. 1e) and, notably, in the indolent/smouldering cases (Fig. 1f; $P=0.048$ ). The performance of the hybrid model at this regard was suboptimal, as it might be anticipated by the combined effects of limited number of cases and events (Fig. 1g, h).

In conclusion, this analysis allowed to confirm the general validity of risk stratification of patients with systemic mastocytosis according to the newly developed Mayo models; furthermore, we revealed its performance in predicting prognosis of intermediate category' patients, especially using the clinical model. The proportionality between risk factors and outcome reaffirms the proof-ofprinciple underneath Mayo model.

The easy and prompt attainability of parameters included in the clinical model makes this particularly suitable for routine patients' management. The use of the hybrid clinical-molecular model might be currently reserved to younger patients, where stem cell transplantation is an 


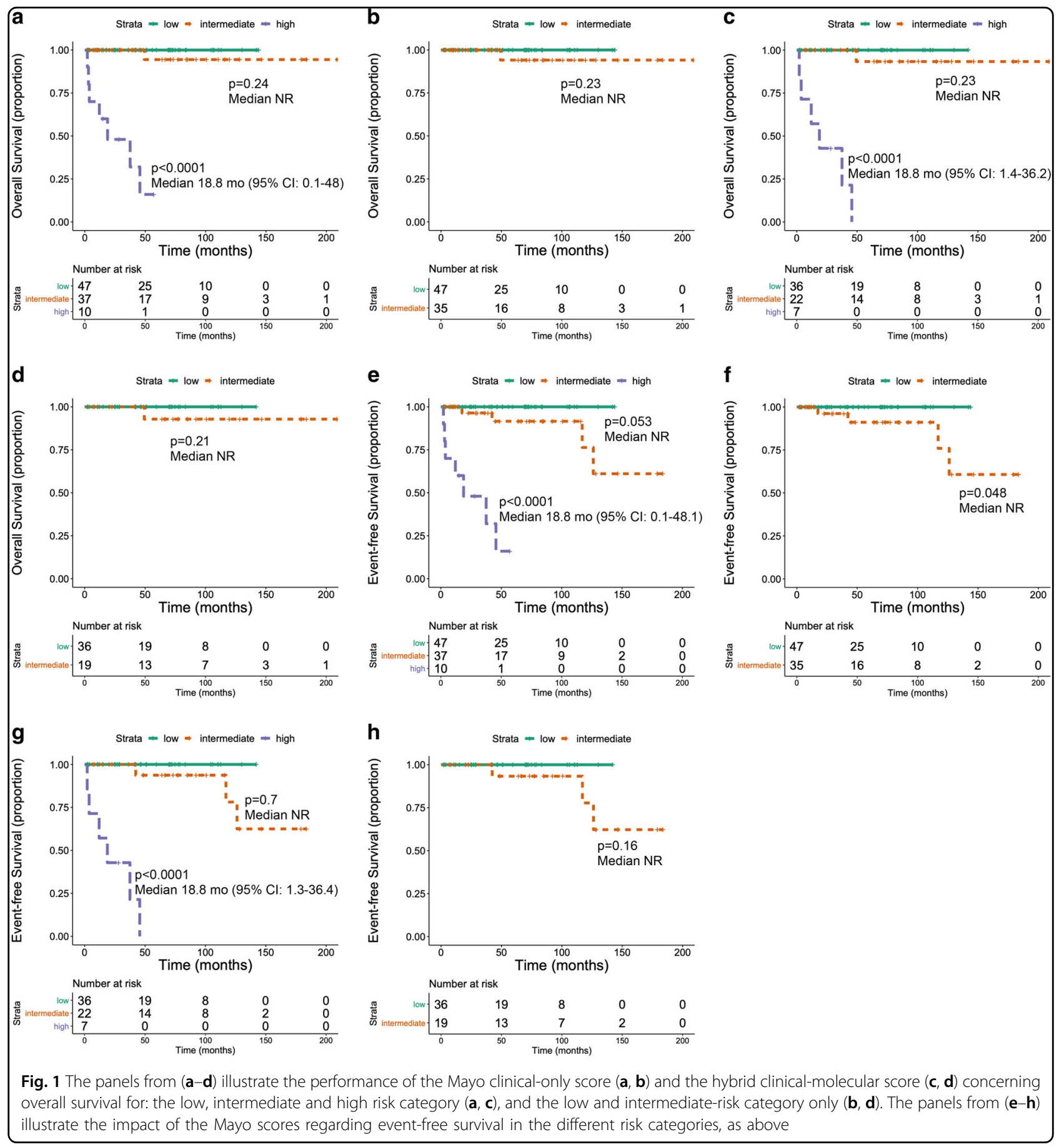

option, and should be definitely incorporated in clinical trials; also, it will be important to prospectively validate its performance in the era of new targeted agents like midostaurin ${ }^{12}$. It is also conceivable that further insights into the mutation landscape of SM patients might lead to improvement of the hybrid clinical-molecular score, and making it more widely available and enhancing its performance.

\section{Acknowledgements}

This work was supported by AIRC $5 \times 1000$ call "Metastatic disease: the key unmet need in oncology" to MYNERVA project, \#21267 (MYeloid NEoplasms Research Venture AIRC). A detailed description of the MYNERVA project is available at http://www.progettoagimm.it. Supported also by a grant from Ministero della Salute GR-2016-02362631 to P.G., Ministero della Salute GR2013-02358238 to F.M., and Ministero della Salute GR-2016-02362631 to L.P.

Conflict of interest

The authors declare that they have no conflict of interest. 


\section{Publisher's note}

Springer Nature remains neutral with regard to jurisdictional claims in published maps and institutional affiliations.

Received: 13 October 2018 Revised: 2 November 2018 Accepted: 6 November 2018

Published online: 11 February 2019

\section{References}

1. Arber, D. A. et al. The 2016 revision to the World Health Organization classification of myeloid neoplasms and acute leukemia. Blood 127, 2391-2405 (2016).

2. Valent, P., Akin, C. \& Metcalfe, D. D. Mastocytosis: 2016 updated WHO classification and novel emerging treatment concepts. Blood 129, 1420-1427 (2017).

3. Pieri, L. et al. Clinical presentation and management practice of systemic mastocytosis. A survey on 460 Italian patients. Am. J. Hematol. 91, 692-699 (2016).

4. Vannucchi, A. M. et al. Mutations and prognosis in primary myelofibrosis. Leukemia 27, 1861-1869 (2013).
5. Guglielmelli, P. et al. MIPSS70: mutation-enhanced international prognostic score system for transplantation-age patients with primary myelofibrosis. J. Clin. Oncol. 36, 301-318 (2018).

6. Pardanani, A. et al. Next-generation sequencing in systemic mastocytosis: derivation of a mutation-augmented clinical prognostic model for survival. Am. J. Hematol. 91, 888-893 (2016).

7. Jawhar, M. et al. Additional mutations in SRSF2, ASXL1 and/or RUNX1 identify a high-risk group of patients with $\mathrm{KIT} \mathrm{D} 816 \mathrm{~V}(+)$ advanced systemic mastocytosis. Leukemia 30, 136-143 (2016).

8. Pardanani, A. D. et al. ASXL1 and CBL mutations are independently predictive of inferior survival in advanced systemic mastocytosis. Br. J. Haematol. 175, 534-536 (2016).

9. Pardanani, A. et al. Mayo alliance prognostic system (MAPS) for mastocytosis: clinical and hybrid clinical-molecular models. Blood Adv. 2, 2964-2972 (2018).

10. Tefferi, A. et al. MIPSS70+Version 2.0: mutation and karyotype-enhanced international prognostic scoring system for primary myelofibrosis. J. Clin. Oncol. 36, JCO2018789867 (2018).

11. Shah, S. et al. Cytogenetic abnormalities in systemic mastocytosis: WHO subcategory-specific incidence and prognostic impact among 348 informative cases.Am. J. Hematol. 93, 1461-1466 (2018).

12. Gotlib, J. et al. Efficacy and safety of midostaurin in advanced systemic mastocytosis. New Engl. J. Med. 374, 2530-2541 (2016). 